\title{
E-MRS Plans 1989 Spring Meeting, Issues Call for Papers
}

The 1989 Spring Meeting of the European Materials Research Society is scheduled for May 30-June 2 at the Council of Europe, Strasbourg, France. Five technical symposia are planned: Third International Symposium on Silicon MBE; Science and Technology of Defects in Silicon; Acoustic, Optical, Thermal Wave Characterization of Material; Beam Processing and Laser Chemistry; and Biomaterials. A course on High Tech Biomaterials in Hard Tissue Repair or Replacement will run parallel to the E-MRS Meeting. [See details in the November MRS BULLETIN, p. 64.]

To obtain information about the meeting or to register, contact the Secretariat, Attention P. Siffert, Centre de Recherches Nucléaires, Laboratoire Phase, F-67037 Strasbourg Cedex, France; telephone 8828 65 43; fax 882809 90; telex 890032 CNRS CRO.

Papers are being solicited for the $1989 \mathrm{E}$ MRS Spring Meeting. The deadline for abstract submission is March 1, 1989. For additional details about symposium topics and abstract submission contact one of the symposium chairs listed below.

\section{Third International Symposium on Silicon MBE}

This symposium will deal with singlecrystal growth, by molecular beam epitaxy, of materials compatible with silicon, their characterization, and device application. Submissions on surface physics and related vacuum synthesis the techniques such as solid phase and ion beam epitaxy are encouraged. Discussions will cover:

- Homoepitaxy-preparation, characterization, alternative growth methods;

- Doping-co-evaporation, implantation, solid phase $\mathrm{MBE}$;

- Heteroepitaxy-nucleation (including III/V on Si), mismatch accommodation, germanium silicon, silicides, insulators;

- Quantum wells and superlatticestheory, properties, ultrathin periods

- Devices-work to date and prospects for exploiting MBE capabilities;

- Apparatus-scaling towards production, in situ monitoring, new advances.

Chairs:

E. Kasper

AEG Research Center

Sedanstr. 10

D-7900 ULM, W. Germany

E.H.C. Parker

University of Warwick

Dept. of Physics

Coventry, CV4 7AL, United Kingdom

\section{Science and Technology of Defects in Silicon}

Topics in this symposium will range from the fundamental characterization of physical properties to the assessment of materials for device applications. Scheduled sessions will span:

- Crystal growth-homo- and heteroepitaxial, SOI, SIMOX;

- Process-induced defects-etching, gettering, heat-treatment, lithography, implantation:

- Topography-imaging, mapping;

- Hydrogenation of silicon-passivation processes;

- Impurities-fast diffusing metals, light and exotic impurities;

- Complexes and interactions between impurities-impurity pairs, thermal donors, multistability.

Chairs:

C.A.J. Ammerlaan

Natuurkundig Laboratorium

University of Amsterdam

Postbus 20215

NL-1000 HE Amsterdam

Netherlands

A. Chantre

Centre National d'Etudes des

Télécommunications

BP 98, F-38243 Meylan Cedex

France

P. Wagner

Heliotronic GmbH, Postfach 1129

D-8263 Burghausen

W. Germany

\section{Acoustic, Optic, Thermal Wave}

Characterization of Material

Chairs:

C.M. Crean

National Microelectronics Research Center

University College, Lee Maltings

Prospect Row

Cork, Ireland

M. Locatelli

Institut de Recherche Fondamentale

Centre d'Etudes Nucléaires de

Grenoble, $85 X$

F-38041 Grenoble Cedex

France

J. McGilp

Trinity College

Dublin, Ireland

\section{Beam Processing and Laser Chemistry}

This symposium will consider both practical and theoretical aspects of energy beam materials processing. A large degree of focus will be given to the use of ion, electron and photon beams, and on laserassisted process chemistry. Thin films, surface and interface reactions, and bulk phase transformations will be emphasized, in addition to practical technological details and the criteria for present and future applications. Topics include:

- Effect of energy beams on surface and interface reactions:

- Bulk and surface transformations induced by energy beams;

- Studies of kinetics and thermodynamics of the stimulated reaction process;

- Deposition, growth and patterning of thin films;

- Applications and technology of energy beams including in situ processing and device manufacturing.

Chairs:

Ian W. Boyd

Dept. of Electronic \& Electrical Engineering

University College London

Torrington Place

London WC1E 7JE

Telephone: $-13877050 \times 3956 \times 7304$

Fax: -13874350

Telex: 296273 UCLENG.G

Emanuele Rimini

Dipartimento di Fisica

Universita di Catania

57 Corso Italia

95129 Catania, Italy

Telephone: -95383446

Fax: -95383023

Telex: 971554 INFNCT

\section{Biomaterials}

Chairs:

D. Muster

L.E.E.D. Biomateriaux

CHRU - BP 426

F-67091 Strasbourg Cedex

France

G. Hastings

Biomedical Engineering Unit - Medical Institute

Hartshill, Stoke-on-Trent

United Kingdom 